\title{
Health Care Analytics With Time-Invariant and Time-Variant Feature Importance to Predict Hospital-Acquired Acute Kidney Injury: Observational Longitudinal Study
}

Horng-Ruey Chua ${ }^{1,2}$, MMED; Kaiping Zheng ${ }^{3}, \mathrm{PhD}$; Anantharaman Vathsala ${ }^{1,2}$, MD; Kee-Yuan Ngiam ${ }^{4,5}$, MMED; Hui-Kim Yap ${ }^{6,7}$, PhD; Liangjian Lu ${ }^{6,7}$, MBBCHIR; Ho-Yee Tiong ${ }^{5,8}$, MMED; Amartya Mukhopadhyay ${ }^{2,9}$, MD; Graeme MacLaren ${ }^{5,10}$, MSc; Shir-Lynn Lim ${ }^{2,11}$, MMED; K Akalya ${ }^{1}$, BSc; Beng-Chin Ooi ${ }^{3}$, PhD

${ }^{1}$ Division of Nephrology, Department of Medicine, National University Hospital, Singapore, Singapore

${ }^{2}$ Department of Medicine, Yong Loo Lin School of Medicine, National University of Singapore, Singapore, Singapore

${ }^{3}$ Department of Computer Science, School of Computing, National University of Singapore, Singapore, Singapore

${ }^{4}$ Division of Endocrine Surgery, Department of Surgery, National University Hospital, Singapore, Singapore

${ }^{5}$ Department of Surgery, Yong Loo Lin School of Medicine, National University of Singapore, Singapore, Singapore

${ }^{6}$ Division of Paediatric Nephrology, Department of Paediatrics, National University Children's Medical Institute, Singapore, Singapore

${ }^{7}$ Department of Paediatrics, Yong Loo Lin School of Medicine, National University of Singapore, Singapore, Singapore

${ }^{8}$ Department of Urology, National University Hospital, Singapore, Singapore

${ }^{9}$ Division of Respiratory and Critical Care Medicine, Department of Medicine, National University Hospital, Singapore, Singapore

${ }^{10}$ Cardiothoracic Intensive Care Unit, Department of Cardiac, Thoracic and Vascular Surgery, National University Hospital, Singapore, Singapore

${ }^{11}$ Department of Cardiology, National University Heart Centre, Singapore, Singapore

\section{Corresponding Author:}

Horng-Ruey Chua, MMED

Division of Nephrology

Department of Medicine

National University Hospital

Level 10 Medicine, NUHS Tower Block

$1 \mathrm{E}$ Kent Ridge Road

Singapore, 119228

Singapore

Phone: 6567726178

Email: horng_ruey chua@nuhs.edu.sg

\section{Abstract}

Background: Acute kidney injury (AKI) develops in 4\% of hospitalized patients and is a marker of clinical deterioration and nephrotoxicity. AKI onset is highly variable in hospitals, which makes it difficult to time biomarker assessment in all patients for preemptive care.

Objective: The study sought to apply machine learning techniques to electronic health records and predict hospital-acquired AKI by a 48-hour lead time, with the aim to create an AKI surveillance algorithm that is deployable in real time.

Methods: The data were sourced from 20,732 case admissions in 16,288 patients over 1 year in our institution. We enhanced the bidirectional recurrent neural network model with a novel time-invariant and time-variant aggregated module to capture important clinical features temporal to AKI in every patient. Time-series features included laboratory parameters that preceded a 48-hour prediction window before AKI onset; the latter's corresponding reference was the final in-hospital serum creatinine performed in case admissions without AKI episodes.

Results: The cohort was of mean age 53 (SD 25) years, of whom 29\%, 12\%, 12\%, and 53\% had diabetes, ischemic heart disease, cancers, and baseline eGFR $<90 \mathrm{~mL} / \mathrm{min} / 1.73 \mathrm{~m}^{2}$, respectively. There were 911 AKI episodes in 869 patients. We derived and validated an algorithm in the testing dataset with an AUROC of $0.81(0.78-0.85)$ for predicting AKI. At a 15\% prediction threshold, our model generated 699 AKI alerts with 2 false positives for every true AKI and predicted $26 \%$ of AKIs. A lowered 5\% prediction threshold improved the recall to $60 \%$ but generated 3746 AKI alerts with 6 false positives for every true AKI. Representative 
interpretation results produced by our model alluded to the top-ranked features that predicted AKI that could be categorized in association with sepsis, acute coronary syndrome, nephrotoxicity, or multiorgan injury, specific to every case at risk.

Conclusions: We generated an accurate algorithm from electronic health records through machine learning that predicted AKI by a lead time of at least 48 hours. The prediction threshold could be adjusted during deployment to optimize recall and minimize alert fatigue, while its precision could potentially be augmented by targeted AKI biomarker assessment in the high-risk cohort identified.

(J Med Internet Res 2021;23(12):e30805) doi: 10.2196/30805

\section{KEYWORDS}

acute kidney injury; artificial intelligence; biomarkers; clinical deterioration; electronic health records; hospital medicine; machine learning

\section{Introduction}

The clinical burden of acute kidney injury (AKI) worsens globally with the increasing complexity of cardiovascular diseases, anticancer therapy, and aging population [1-3]. AKI develops in $4 \%$ of patients admitted to our institution and involves more than 3000 patients annually [4]. A total of $39 \%$ of AKI cases develop during hospitalization following clinical deterioration and multiorgan dysfunction [4,5]. Additionally, $15 \%$ of patients who receive antimicrobials or chemotherapy of nephrotoxic potential develop drug-induced AKI [6,7]. Iodinated contrast administered for angiography contributes to AKI in $10 \%$ to $40 \%$ of patients with chronic kidney disease $[8,9]$. Once AKI develops in patients, however, the management remains supportive with control of its underlying triggers. AKI portends a poor patient prognosis with high mortality, prolonged hospitalization, and sustained deterioration of kidney function, with a significant risk of kidney failure in the long term [10,11].

Management strategies for high-risk patients may prevent AKI or reduce its downstream complications should AKI still develop. These measures must be implemented promptly, which requires the diagnosis of $\mathrm{AKI}$ in the subclinical phase, way before its onset. As the onset of AKI is highly variable during a patient's stay, it is unclear how best to time biomarker surveillance for kidney injury concerning the patient's clinical progress. The advent of electronic health records (EHRs) now provides us with real-time clinical data from routine patient care, built into millions of data points for analytics. These, along with AKI being defined by a numerical measure using serial serum creatinine, allow for an AKI prediction algorithm that is reproducible on a large scale. Machine learning with recurrent neural network-based techniques could improve the accuracy of analytics over traditional biostatistics [12]. These could be enhanced by capturing the relative feature importance temporal to AKI; that is, certain clinical covariates or trends (ie, features) would factor with increasing (or decreasing) importance in the time leading up to the onset of AKI. In this study, we would apply a novel machine learning technique that analyzes patient-related features in the form of routine hematology and biochemistry and their interaction with time to accurately predict AKI in hospitals by a lead time of 48 hours.

\section{Methods}

\section{Dataset}

The data source was our institution's EHR in 2012, which recorded clinical and laboratory data from 68,832 case admissions in that year. Our institution is a 1200-bed academic hospital that provides complex tertiary care services including cardiothoracic surgery, transplantation, and cancer management. The Institutional Human Research Ethics Committee approved the study (NUHS-DSRB 2018/00169) and waived the need for informed consent given the use of deidentified data for analytics with secured institutional governance.

\section{Study Design and Participants}

We performed an observational longitudinal study of the prospectively acquired EHR data from hospitalized patients in 2012. The exclusion criteria were (1) patients discharged within 48 hours of admission; (2) patients with community-acquired AKI, as inferred from onset of AKI within 48 hours of hospitalization [13]; (3) patients with stage 5 chronic kidney disease by Kidney Disease: Improving Global Outcomes (KDIGO) criteria, both dialysis, and nondialysis [14], inferred from diagnosis codes (Systematized Nomenclature of Medicine-Clinical Terms) for "end-stage kidney/renal disease," an admission estimated glomerular filtration rate (eGFR) of less than $15 \mathrm{~mL} / \mathrm{min} / 1.73 \mathrm{~m}^{2}$ by Chronic Kidney Disease Epidemiology Collaboration equation [15], or procedural codes for peritoneal dialysis catheter insertion, arteriovenous access creation, or fistuloplasty; (4) patients with procedural codes for “**dialysis," “**filtration," or “**diafiltration” previously who failed to recover kidney function to a current admission eGFR of at least $30 \mathrm{~mL} / \mathrm{min} / 1.73 \mathrm{~m}^{2}$; or (5) patients with no available laboratory results for analytics.

\section{Definition of AKI}

The binary event measure was AKI, as defined by the KDIGO 2012 criteria using serial serum creatinine levels during the index hospitalization [16]. These included the relative criterion of at least 1.5 times an increase in serum creatinine level within a 7-day window; the absolute criterion was an increase in serum creatinine of greater than $26.5 \mu \mathrm{mol} / \mathrm{L}(0.3 \mathrm{mg} / \mathrm{dL})$ within 48 hours. The reference serum creatinine within the corresponding 7-day or 48-hour window for either criterion was taken as the baseline creatinine. The AKI-defining creatinine level and the extent of elevation over baseline were used to grade the initial 
KDIGO AKI staging severity. Creatinine was measured using the ADVIA 2400 (Siemens AG) enzymatic method traceable to isotope dilution mass spectrometry standard. We did not apply the oliguria criterion for AKI.

\section{Features Used for Analytics}

Features (or covariates) were sourced from time-series laboratory results. The data source was our institution's EHR, Computerized Patient Support System version 2 (Integrated
Health Information System Pte Ltd). The results were integrated from comma-separated value files using common masked identifiers and ported onto our institution's artificial intelligence discovery platform, an EHR analytic module. Data with the date and time stamps were selected as features to predict the event. These included all serial hematology, serum biochemistry, and urinary investigations (Figure 1). We did not include disease diagnosis codes or medication records.

Figure 1. Prediction logic and features included in analytics. *: Serum biochemistry or hematology unless otherwise stated (eg, urine WBC and RBC); **: AKI defined by KDIGO criteria; $\mathrm{x}$ : features entered in model; $\mathrm{t}$ : time windows; $\beta$ : time-invariant feature importance of which influence is shared across time windows; alpha-t: time-variant feature importance; ht: time-variant hidden representation; WBC: white blood cell; RBC: red blood cell; AKI: acute kidney injury; KDIGO: Kidney Disease: Improving Global Outcomes; BIRNN: bidirectional recurrent neural network; FiLM: feature-wise linear modulation.

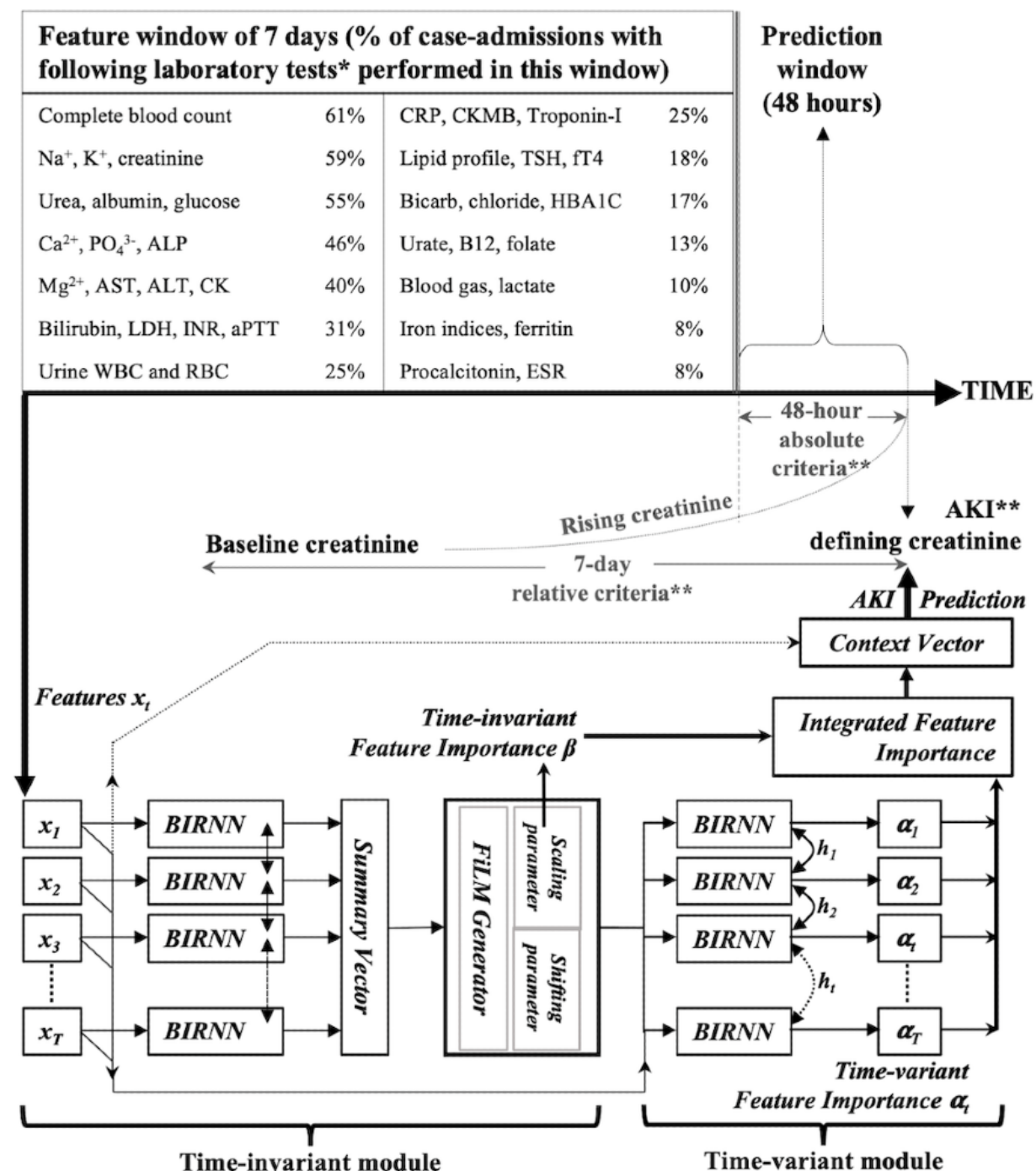

\section{Analytics}

Patient profile was compared between unique patients who developed AKI and those who did not. Parametric variables were reported as mean and standard deviation and compared using Student $t$ tests; nonparametric variables were reported as median and IQR and compared using Wilcoxon rank-sum tests. Categorical variables were reported as frequency and percentage and compared using chi-square or Fisher exact tests where appropriate. A 2-tailed $P$ value of $<.05$ was taken as the measure of statistical significance.

We sectioned the dataset by date and time for predictive analytics. Every case admission was taken as one sample. The first AKI episode that occurred in corresponding case admissions was analyzed. The AKI-defining creatinine served as the reference time point; the immediately preceding 48 hours was made the prediction window, and the feature window included the time up to 7 days before the prediction window (Figure 1). For case admissions with no AKI episodes, the corresponding 
reference time point would be the final serum creatinine level and likewise preceded by a 48-hour prediction window and a further 7-day feature window. Features performed within the feature window were used to predict AKI, a bivariate event, by a lead time of 48 hours. The feature window was further sectioned into daily serial time intervals for time-series modeling, temporal to the event. For each time interval, we averaged the values of the same feature, followed by normalization of the corresponding result $x$ to generate a normalized $x^{1}$ as the input for analytics, where $x^{1}=[x-$ $\operatorname{minimum}(x)] /[\operatorname{maximum}(x)-\operatorname{minimum}(x)]$.

We proposed a novel time-invariant and time-variant (TITV) model to facilitate more accurate and interpretable analytics in AKI prediction based on the collaboration of 3 modules [17] (Figure 1). In the time-invariant module, an abstract representation was calculated with the data in the entire feature window, denoting each feature's importance shared across time (ie, time-invariant feature importance). This time-invariant feature importance guided the modulation of input in the next module, the time-variant module. In this second module, we applied a bidirectional recurrent neural network to process sequential data and capture the dynamic behavior both forward and backward in time temporal to the event, as guided by the computed time-invariant feature importance from the time-invariant module. Additionally, we differentiated the influence of features across time windows leading to the event by applying the self-attention mechanism on top of the output of the bidirectional recurrent neural network; the output after the self-attention mechanism represents each feature's importance in the corresponding time window (ie, time-variant feature importance in this time-variant module). Finally, in the prediction module, both the time-invariant and the time-variant feature importance were aggregated to calculate the final prediction (ie, risk of AKI). Meanwhile, the influence of each feature (in each time window) on the final prediction was also derived from the TITV model.

We performed a random shuffling of the entire cohort and arbitrarily partitioned the samples into $80 \%$ training, $10 \%$ validation, and $10 \%$ testing datasets. In the training process, we selected the hyperparameters that achieved the best performance in the validation dataset and applied them to the testing dataset for reporting of the experimental results [18-20]. We examined the reporting performance using the area under the receiver operating characteristic curve (AUC), as well as the respective sensitivity (recall) and positive predictive values (precision) that corresponded with the varying model prediction thresholds for AKI. Precision represents the proportion of predicted cases that truly had AKI; recall represents the proportion of actual AKI cases successfully identified by the prediction model. The AKI prediction threshold that provided the most optimal statistical balance between precision and recall was inferred by the highest computed F1 score. A high model recall gives rise, however, to more false positives (ie, poorer precision), and these permutations were further examined to demonstrate their clinical relevance to AKI diagnostics. These results were compared with the corresponding performance using traditional logistic regression and baseline recurrent neural network models. We applied zero imputation for missing data. Analysis was performed using Python (version 3.8.2, open source for Mac OSX).

\section{Results}

\section{Patient Profile}

We studied 20,732 case admissions in 16,288 unique patients, of which 1971 patients were younger than age 18 years (Figure 2 ). The mean age of the final cohort was 53 (SD 25) years, and $52.2(8510 / 16,288)$ were males; $28.9 \%(4701 / 16,288)$ had diabetes, $35.0 \%(5699 / 16,288)$ had hypertension, $11.7 \%$ $(1898 / 16,288)$ had ischemic heart disease, and $11.7 \%$ $(1899 / 16,288)$ had either solid organ or hematological malignancy. Near half $(7214 / 16,288,44.3 \%)$ of patients had a baseline eGFR $<90 \mathrm{~mL} / \mathrm{min} / 1.73 \mathrm{~m}^{2}$. More patients with AKI $(258 / 869,29.7 \%)$ had a baseline eGFR $<60 \mathrm{~mL} / \mathrm{min} / 1.72 \mathrm{~m}^{2}$ compared to those without AKI $(2738 / 15,419,17.8 \% ; P<.001$; Table 1). 
Figure 2. Study flow diagram. AKI: acute kidney injury; CKD: chronic kidney disease; CKD-EPI: Chronic Kidney Disease Epidemiology Collaboration equation; eGFR: estimated glomerular filtration rate; ESKD: end-stage kidney disease; RRT: renal replacement therapy.

\begin{tabular}{|c|c|}
\hline 68,832 inpatient cases in 2012 & Exclusion criteria \\
\hline & $\begin{array}{l}40,044 \text { cases admitted for } 48 \text { hours or less. } \\
\text { (Exclusion-i) }\end{array}$ \\
\hline & $\begin{array}{l}864 \text { cases of community-acquired AKI. } \\
\text { (Exclusion-ii) }\end{array}$ \\
\hline & $\begin{array}{l}1,630 \text { cases with CKD stage } 5 \text { or ESKD-related } \\
\text { diagnosis or chronic dialysis procedural codes. }\end{array}$ \\
\hline & $\begin{array}{l}179 \text { cases with admission } \mathrm{CKD} \text {-EPI } \text { eGFR }<15 \\
\mathrm{~mL} / \mathrm{min} / 1.73 \mathrm{~m}^{2} . \\
\text { (Exclusion-iii) }\end{array}$ \\
\hline & $\begin{array}{l}1 \text { case with prior RRT and current admission } \\
\text { eGFR }<30 \mathrm{~mL} / \mathrm{min} / 1.73 \mathrm{~m}^{2} \text {. } \\
\text { (Exclusion-iv) }\end{array}$ \\
\hline $\begin{array}{l}\text { 26,114 inpatient cases: } \\
\cdot \quad 960 \text { AKI cases }-3.7 \% \\
\cdot \quad 25,154 \text { non-AKI cases }-96.3 \%\end{array}$ & \\
\hline & $\begin{array}{l}5,382 \text { cases without laboratory results in the } \\
\text { feature window. } \\
\text { (Exclusion-v) }\end{array}$ \\
\hline \multicolumn{2}{|c|}{$\begin{array}{l}\text { 20,732 inpatient cases (in 16,288 unique patients) in final analysis: } \\
\text {. } 911 \text { AKI cases }-4.4 \% \text {; in } 869 \text { unique patients. } \\
\text { - } 19,821 \text { non-AKI cases }-95.6 \% \text {; in } 15,419 \text { unique patients. }\end{array}$} \\
\hline
\end{tabular}


Table 1. Study profile and bivariate comparison between acute kidney injury and non-acute kidney injury patients.

\begin{tabular}{|c|c|c|c|c|}
\hline Variables & Entire cohort $(\mathrm{n}=16,288)$ & $\mathrm{AKI}^{\mathrm{a}}(\mathrm{n}=869)$ & Non-AKI $(\mathrm{n}=15,419)$ & $P$ value \\
\hline Age, mean (SD), years & $53(25)$ & $62(22)$ & $53(26)$ & $<.001$ \\
\hline Male gender, $\mathrm{n}(\%)$ & $8510(52.2)$ & $480(55.2)$ & $8030(52.1)$ & .08 \\
\hline \multicolumn{5}{|l|}{ Comorbidities, n (\%) } \\
\hline Diabetes & $4701(28.9)$ & $371(42.7)$ & $4330(28.1)$ & $<.001$ \\
\hline Hypertension & $5699(35.0)$ & $498(57.3)$ & $5201(33.7)$ & $<.001$ \\
\hline Ischemic heart disease & $1898(11.7)$ & $262(30.1)$ & $1636(10.6)$ & $<.001$ \\
\hline Heart failure & $1138(7.0)$ & $190(21.9)$ & $948(6.1)$ & $<.001$ \\
\hline Cerebrovascular disease & $757(4.6)$ & $73(8.4)$ & $684(4.4)$ & $<.001$ \\
\hline Chronic liver disease & $269(1.7)$ & $44(5.1)$ & $225(1.5)$ & $<.001$ \\
\hline Solid organ malignancy & $1556(9.6)$ & $140(16.1)$ & $1416(9.2)$ & $<.001$ \\
\hline Hematological malignancy & $343(2.1)$ & $52(6.0)$ & $291(1.9)$ & $<.001$ \\
\hline \multicolumn{5}{|l|}{ Baseline kidney function } \\
\hline Creatinine, $\mu \mathrm{mol} / \mathrm{L}$, median (IQR) & $71(54-92)$ & $69(46-108)$ & $71(55-91)$ & .05 \\
\hline $\mathrm{eGFR}^{\mathrm{b}}, \mathrm{mL} / \mathrm{min} / 1.73 \mathrm{~m}^{2}$, median (IQR) & $91(67-109)$ & $86(55-110)$ & $91(68-109)$ & $<.001$ \\
\hline eGFR 90 or above $\mathrm{mL} / \mathrm{min} / 1.73 \mathrm{~m}^{2}, \mathrm{n}(\%)$ & $7628(46.8)$ & $400(46.0)$ & $7228(46.9)$ & .65 \\
\hline eGFR 60 to $<90 \mathrm{~mL} / \mathrm{min} / 1.73 \mathrm{~m}^{2}, \mathrm{n}(\%)$ & $4218(25.9)$ & $211(24.3)$ & $4007(26.0)$ & .28 \\
\hline eGFR 45 to $<60 \mathrm{~mL} / \mathrm{min} / 1.73 \mathrm{~m}^{2}, \mathrm{n}(\%)$ & $1328(8.2)$ & $101(11.6)$ & $1227(8.0)$ & $<.001$ \\
\hline eGFR 30 to $<45 \mathrm{~mL} / \mathrm{min} / 1.73 \mathrm{~m}^{2}, \mathrm{n}(\%)$ & $950(5.8)$ & $90(10.4)$ & $860(5.6)$ & $<.001$ \\
\hline $\mathrm{eGFR}<30 \mathrm{~mL} / \mathrm{min} / 1.73 \mathrm{~m}^{2}, \mathrm{n}(\%)$ & $718(4.4)$ & $67(7.7)$ & $651^{\mathrm{c}}(4.2)$ & $<.001$ \\
\hline \multicolumn{5}{|l|}{ AKI-related variables } \\
\hline AKI-defining creatinine, $\mu \mathrm{mol} / \mathrm{L}$, median $(\mathrm{IQR})$ & $-{ }^{\mathrm{d}}$ & $122(80-169)$ & - & - \\
\hline Relative criterion (vs absolute), $\mathrm{n}(\%)$ & - & $651(74.9)$ & - & - \\
\hline AKI onset days from admission, median (IQR) & - & $6(3-10)$ & - & - \\
\hline \multicolumn{5}{|l|}{ Serum biochemistry at AKI detection, median (IQR) } \\
\hline Sodium, $\mathrm{mmol} / \mathrm{L}$ & - & $138(135-142)$ & - & - \\
\hline Potassium, mmol/L & - & $4.1(3.7-4.6)$ & - & - \\
\hline Urea, $\mathrm{mmol} / \mathrm{L}$ & - & $11(7-15)$ & - & - \\
\hline Bicarbonate, $\mathrm{mmol} / \mathrm{L}$ & - & $24(19-27)$ & - & - \\
\hline Phosphate, mmol/L & - & $1.23(0.95-1.54)$ & - & - \\
\hline Calcium, mmol/L & - & $2.03(1.89-2.17)$ & - & - \\
\hline Chloride, $\mathrm{mmol} / \mathrm{L}$ & - & $105(101-109)$ & - & - \\
\hline Uric acid, $\mu \mathrm{mol} / \mathrm{L}$ & - & $384(266-527)$ & - & - \\
\hline \multicolumn{5}{|l|}{ Initial KDIGO ${ }^{\mathrm{e}}$ AKI staging, n (\%) } \\
\hline Stage 1 & - & $701(80.7)$ & - & - \\
\hline Stage 2 & - & $125(14.4)$ & - & - \\
\hline Stage 3 & - & $43(4.9)$ & - & - \\
\hline Total cumulative hospital days, median (IQR) & $5(3-10)$ & $23(13-44)$ & $5(3-9)$ & $<.001$ \\
\hline Hospital days per admission, median (IQR) & $5(3-8)$ & $14(8-26)$ & $5(3-7)$ & $<.001$ \\
\hline
\end{tabular}

${ }^{\mathrm{a}} \mathrm{AKI}$ : acute kidney injury.

b eGFR: estimated glomerular filtration rate by Chronic Kidney Disease Epidemiology Collaboration equation. 
${ }^{\mathrm{c}} \mathrm{A}$ total of 1446 non-AKI patients had missing baseline eGFR.

${ }^{\mathrm{d}}$ Not applicable.

${ }^{\mathrm{e}}$ KDIGO: Kidney Disease: Improving Global Outcomes.

\section{Evaluation Outcomes}

AKI developed during 4.4\% (911/20,732) of case admissions in 869 unique patients at a median of 6 (IQR 3-10) days from admission; 74.9\% (651/869) of AKI patients were diagnosed based on KDIGO relative criterion, and $80.7 \%$ (701/869) were of initial KDIGO stage 1 in severity. Patients who developed AKI were older with more comorbidities including diabetes, hypertension, cardiovascular diseases, chronic kidney disease, chronic liver disease, and cancers compared with non-AKI patients (all $P<.001$ ). The median hospital days per admission and cumulatively in 2012 in AKI patients versus those without were 14 (IQR 8-26) days versus 5 (IQR 3-7) days, and 23 (IQR 13-44) days versus 5 (IQR 3-9) days, respectively (all $P<.001$; Table 1).

\section{Analytics for AKI Prediction in the Hospital}

The 7-day feature window was divided into daily time windows, giving a total of 7 time windows and 709 features in the analysis. Figure 1 shows the laboratory variables included in the feature window in order of their corresponding test prevalence by categories. Complete blood count was the most common investigation, performed in $61.3 \%(12,709 / 20,732)$ of all case admissions in the analysis; this was followed by serum electrolytes, urea, and creatinine at $46 \%$ to $59 \%$, and liver function markers at $30 \%$ to $41 \%$. In comparison, acid-base parameters and serum lactate contributed less $(2146 / 20,732$, $10.4 \%)$ to the analysis.

The cohort was partitioned into the training (16,585 cases), validation (2073 cases), and testing (2074 cases) datasets; AKI rates in the 3 datasets were $4.5 \%, 3.9 \%$, and $4.3 \%$, respectively. Table 2 summarizes the AUC of respective analytic modules in the final testing dataset as well as the precision and recall corresponding with the AKI prediction threshold with the highest F1 score. The AUC for AKI prediction by the multivariate logistic regression and recurrent neural network/time-series models were $79 \%$ and $80 \%$, respectively. The AUC was $81 \%$ after we applied the TITV module with comparable precision and recall compared with the former models; these and the highest F1 score were achieved at an AKI prediction threshold between $15 \%$ and $20 \%$. The respective AUCs and corresponding area under precision-recall curves for the training and testing datasets are illustrated in Figure 3.

Table 3 shows the breakdown in our TITV module precision and recall according to the varying probability thresholds for AKI prediction.

A low prediction threshold detected a very high number of predicted AKI cases that scored high in recall but poor in discrimination between true and false positives. Conversely, a high prediction threshold detected a low number of predicted AKI cases but with high precision. A 15\% AKI probability threshold implied that 699 cases were predicted to be diagnosed with AKI; 33.3\% (233/699) of predicted cases did subsequently develop AKI, while 25.6\% (233/911) of eventual AKI cases were successfully predicted. Reducing the probability threshold to 5\% led to 3746 predicted AKI cases with much higher false positives but with successful prediction of 60.0\% (547/911) of eventual AKI cases. Figure 4 illustrates the confusion matrix plots at AKI prediction thresholds of 5\% and $15 \%$. Further details on TITV performance metrics are provided in Table 4.

In addition, our TITV model generated representative interpretation results specific to each AKI case. Figure 5 illustrates the relative feature importance to AKI in 8 case examples, which demonstrated the range of inflammatory, cardiac, drug-specific, or hepatic functional markers in association with AKI, specific to each case. The source codes for our predictive algorithm are available online [21].

Table 2. Acute kidney injury predictive performance in the testing dataset with optimized F1.

\begin{tabular}{lllll}
\hline Model & Precision $^{\mathrm{a}}$ & Recall $^{\mathrm{b}}$ & $\mathrm{F}^{\mathrm{c}}$ & $\mathrm{AUC}^{\mathrm{d}}(95 \% \mathrm{CI})$ \\
\hline Logistic regression & 0.274 & 0.189 & 0.224 & $0.789(0.752-0.827)$ \\
$\mathrm{RNN}^{\mathrm{e}}\left(\mathrm{GRU}^{\mathrm{f}}\right)$ & 0.286 & 0.222 & 0.250 & $0.800(0.764-0.836)$ \\
$\mathrm{BRNN}^{\mathrm{g}}\left(\mathrm{BGRU}^{\mathrm{h}}\right)$ & 0.309 & 0.233 & 0.266 & $0.797(0.761-0.833)$ \\
Proposed $^{\mathrm{TITV}}{ }^{\mathrm{i}}$ model & 0.397 & 0.256 & 0.311 & $0.814(0.780-0.848)$ \\
\hline
\end{tabular}

${ }^{\mathrm{a}}$ Precision: true positive / (all cases predicted at risk of acute kidney injury).

${ }^{b}$ Recall: true positive / (all cases that eventually developed acute kidney injury).

${ }^{\mathrm{c}}$ F1 score: $2 \times[$ (recall $\times$ precision $) /($ recall + precision $\left.)\right]$.

d AUC: area under receiver operating characteristic curve.

${ }^{\mathrm{e}} \mathrm{RNN}$ : recurrent neural network.

${ }^{\mathrm{f}} \mathrm{GRU}$ : gated recurrent unit.

${ }^{\mathrm{g}}$ BRNN: bidirectional recurrent neural network.

${ }^{\mathrm{h}}$ BGRU: bidirectional gated recurrent unit.

${ }^{\mathrm{i}}$ TITV: time-invariant and time-variant feature importance. 
Figure 3. Area under receiver operating characteristic and area under precision-recall curves of training and testing datasets. AUC: area under receiver operating characteristic curve; AUPRC: area under precision-recall curve.
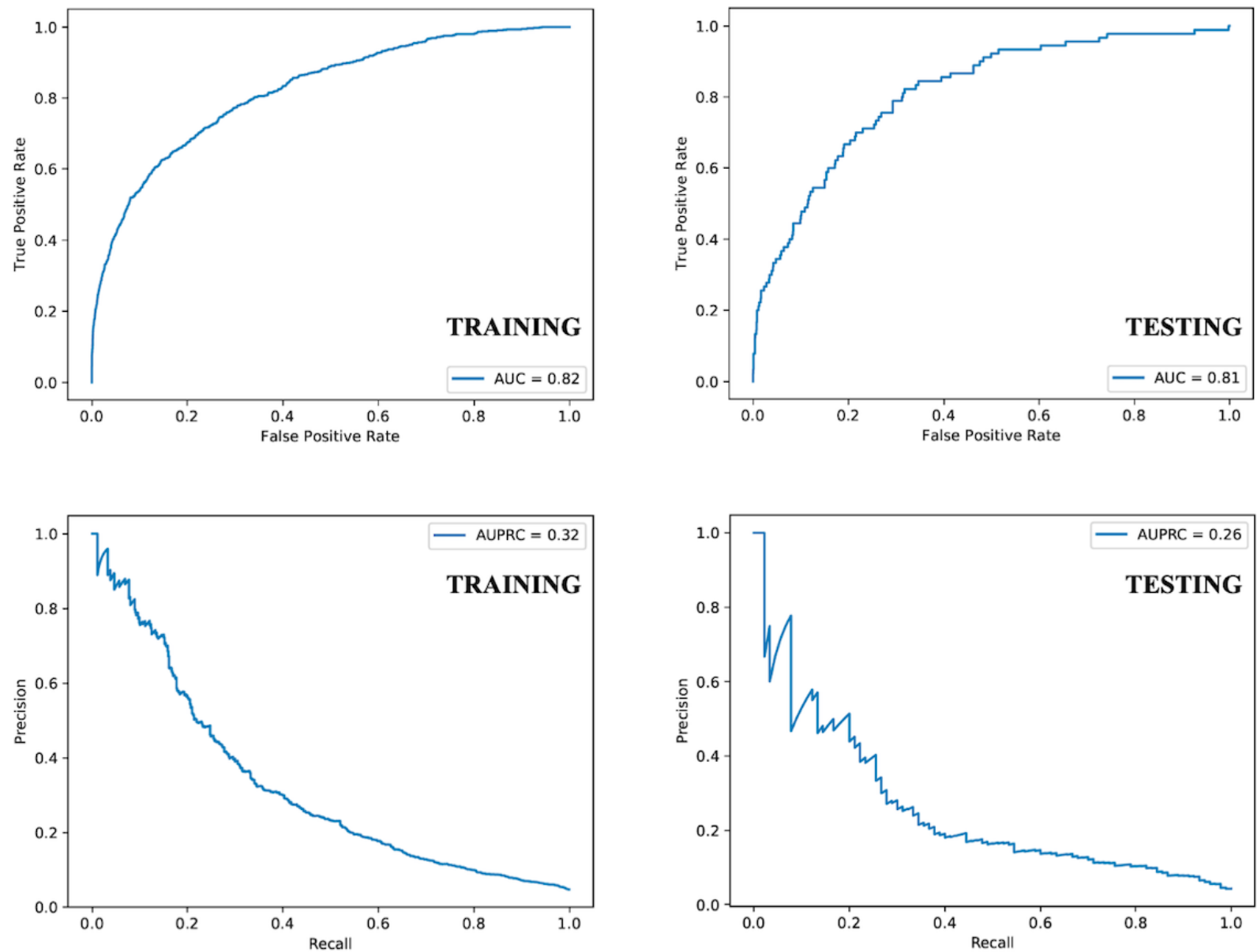

Table 3. Varied acute kidney injury prediction thresholds on time-invariant and time-variant model performance metrics.

\begin{tabular}{llllll}
\hline Threshold $^{\mathrm{a}}$ to predict AKI $^{\mathrm{b}}(\%)$ & Precision $^{\mathrm{c}}$ & Recall $^{\mathrm{d}}$ & $\mathrm{F}^{\mathrm{e}}$ & Predicted AKI cases by model, $\mathrm{n}^{\text {True positive AKI cases, } \mathrm{n}}$ \\
\hline 5 & 0.146 & 0.600 & 0.235 & 3746 & 547 \\
10 & 0.252 & 0.333 & 0.287 & 1204 & 304 \\
15 & 0.333 & 0.256 & 0.289 & 699 & 233 \\
20 & 0.500 & 0.200 & 0.286 & 364 & 182 \\
25 & 0.480 & 0.133 & 0.209 & 253 & 121 \\
30 & 0.556 & 0.111 & 0.185 & 182 & 101 \\
\hline
\end{tabular}

${ }^{a}$ Probability threshold to define predicted AKI versus no risk of AKI (ie, positive/negative class prediction). A low threshold risks over-detection and alert fatigue, which corresponds to poor precision. A high threshold risks missing true AKI cases, which corresponds to poor recall.

${ }^{\mathrm{b}} \mathrm{AKI}$ : acute kidney injury.

${ }^{\mathrm{c}}$ Precision: true positive / (all cases predicted at risk of AKI).

${ }^{\mathrm{d}}$ Recall: true positive / (all cases who eventually developed AKI).

${ }^{\mathrm{e}} \mathrm{F} 1$ score: $2 \times[($ recall $\times$ precision $) /($ recall + precision $)]$. 
Figure 4. Confusion matrix plots with acute kidney injury prediction thresholds at 5\% and $15 \%$.

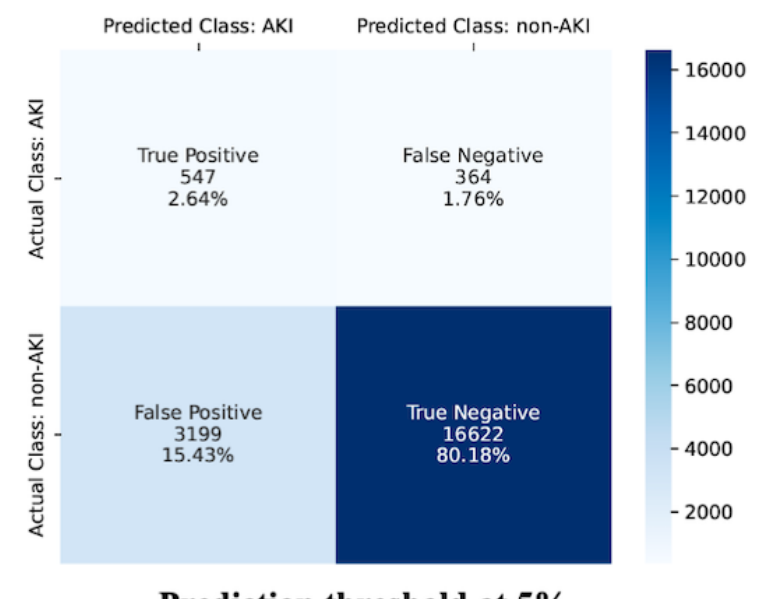

Prediction threshold at $5 \%$

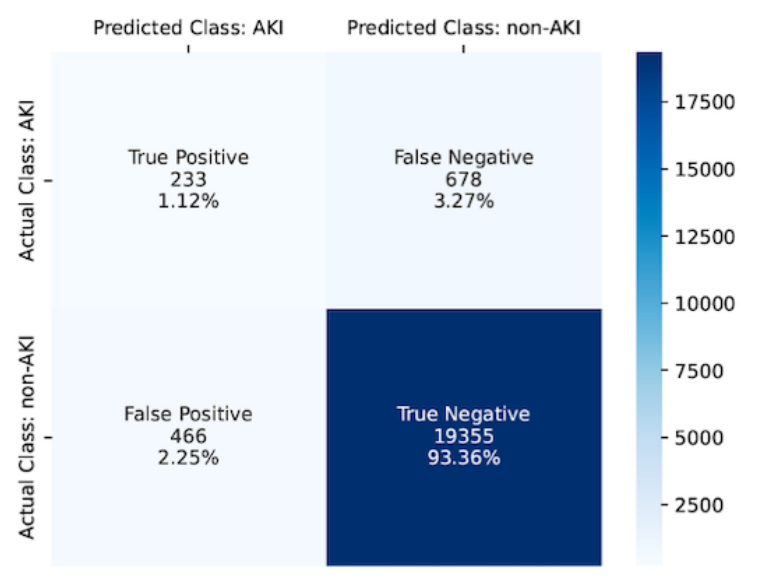

Prediction threshold at $\mathbf{1 5 \%}$

Table 4. Model performance metric with time-invariant and time-variant prediction thresholds at $5 \%$ and $15 \%$.

\begin{tabular}{llll}
\hline & True AKI ${ }^{\mathrm{a}}$ cases & No AKI & Subtotal \\
\hline $\mathbf{5 \%}$ prediction threshold & 547 & 3199 & 3746 \\
TITV $^{\mathrm{b}}$ predicted (positive) & 364 & 16,622 & 16,986 \\
TITV predicted (negative) & 911 & 19,821 & 20,732 \\
Subtotal & & & 699 \\
$\mathbf{1 5 \%}$ prediction threshold & 233 & 466 & 20,033 \\
TITV predicted (positive) & 678 & 19,355 & 20,732 \\
TITV predicted (negative) & 911 & 19,821 & \\
Subtotal & &
\end{tabular}

${ }^{\mathrm{a}} \mathrm{AKI}$ : acute kidney injury.

b TITV: time-invariant and time-variant module. 
Figure 5. Case examples of relative feature importance in acute kidney injury (AKI) prediction. Time-window: refers to feature window of 7 days in AKI prediction; Y-axis: features highly associated with AKI would rank high in relative feature importance; a-b: C-reactive protein, neutrophils featured prominently over days, which suggested infection and inflammation were associated with subsequent AKI; c-d: troponin-I featured prominently initially, which suggested cardiac disease in association with AKI, although its relative importance waned in subsequent days; e-f: vancomycin levels rose in feature importance proximate to AKI, which strongly suggested vancomycin nephrotoxicity; g-h: lactate, liver enzymes, international normalized ratio, and activated partial thromboplastin time featured strongly, which suggested hepatic or multiorgan dysfunction in association with evolving AKI.
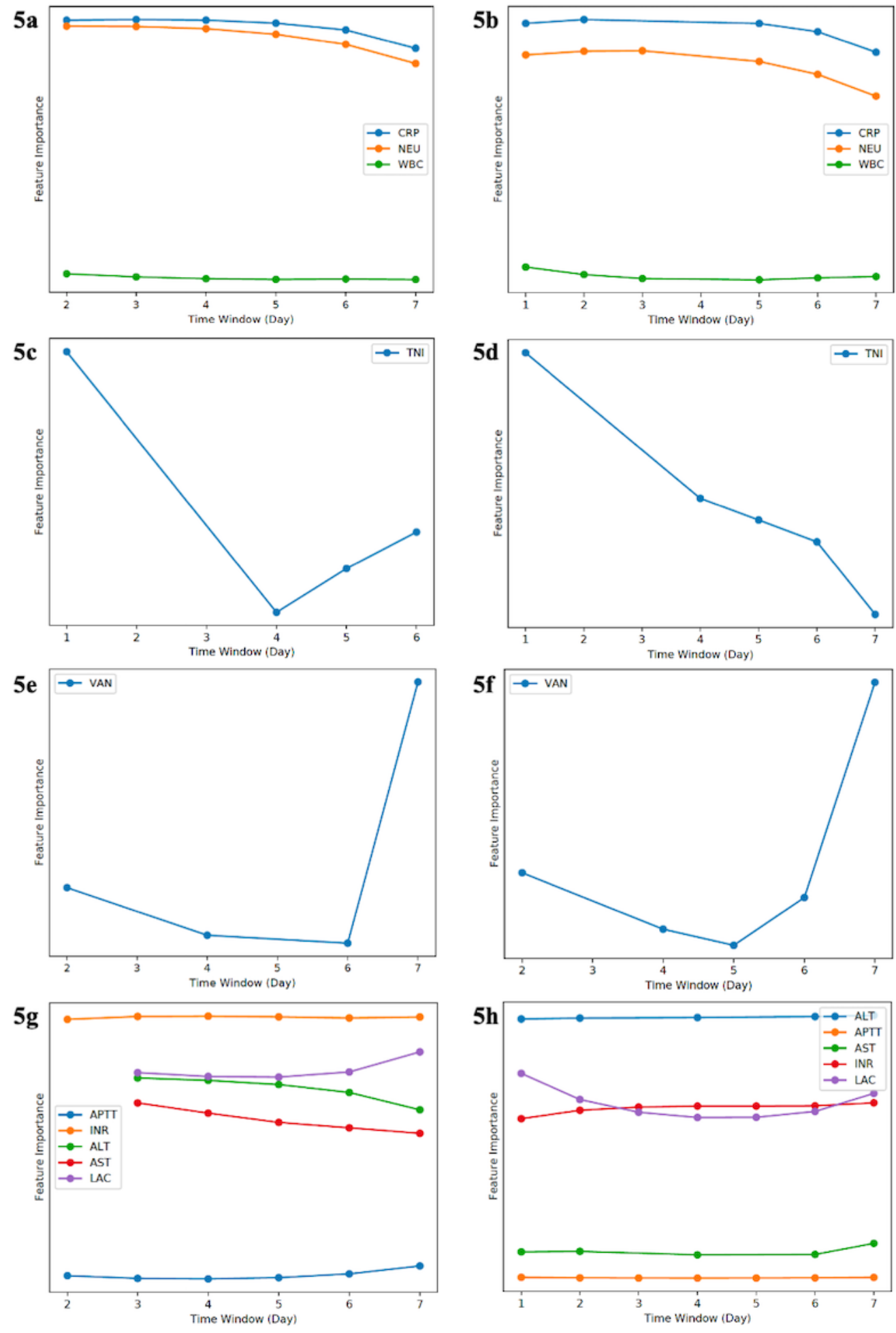


\section{Discussion}

\section{Principal Findings}

We have used structured but heterogeneous biochemical data from 20,732 case admissions in the prediction of hospital-acquired AKI by a 48-hour lead time. We enhanced the recurrent neural network model with a novel analytic module that took into account the temporal interactions in serial laboratory parameters that inferred disease trajectory leading up to AKI [17]. At the optimal statistical operation point as indicated by the highest F1 score (Table 2), our module generated 3 false positives for every 2 true AKI cases, and clinicians would need to act on just 600 predicted AKI alerts of 20,732 case admissions yearly; however, 3 of 4 true AKI cases would be missed. It may be more desirable for our module to successfully predict at least 3 of 5 true AKI cases, but this is counterbalanced by 6 false positives for every 1 true AKI case, and more than 3000 predicted AKI alerts yearly (Table 3). We suggest that our AKI prediction threshold should be low to identify more patients at risk of AKI daily. This narrows the entire hospital cohort to a more manageable patient number for closer monitoring, in whom further assessment could be augmented by AKI biomarkers to reduce false positives [22]. These include urinary clusterin, kidney injury molecule-1, tissue inhibitor of metalloproteinase-2, and insulin-like growth factor binding protein-7, for which levels rise in 12 to 48 hours before a significant rise in serum creatinine [7,23].

\section{Comparison With Prior Work}

Our methodology differs from machine learning techniques that used a quasi-random selection of variable prediction points relative to AKI [24]. It resembles models that adopted structured feature and prediction windows relative to AKI that facilitate the deployment of our prediction algorithm in real time [25]. Importantly, we expanded the prediction window to a minimum of 48 hours. Such improved lead time may be necessary for any AKI preventive strategies to make a meaningful change in clinical outcomes. Preemptive interventions may include more detailed patient reviews, timely treatment of infections, precise volume management [26], preferred use of balanced electrolyte over chloride-rich solutions [27], admission to high-dependency or intensive care unit for detailed monitoring, and reduction in or cessation of nephrotoxic medications [28]. These measures, when implemented in a timely fashion and supported by a responsive EHR platform for AKI alerts, may reduce the hospital days and AKI duration in affected patients [29,30].

The performance of any analytic module depends strongly on the appropriate feature selection. Our model was built from objective laboratory test results that would be similar in data structure across institutions [31]. Our algorithm used routinely performed hematology and biochemistry without disease diagnosis codes; these included complete blood count, common electrolytes, acid-base parameters, and liver and cardiac enzymes, and these remain relevant for current AKI prediction even with the changing health care landscape. As our analysis was limited to available investigations performed before a mandatory 48-hour prediction window, the laboratory indices analyzed in the feature window might not be comprehensive.
This could compromise the model performance, and the prediction should otherwise improve with features performed at higher frequency and more proximate to AKI $[25,32]$. Despite this, we demonstrated an AUC that exceeded $80 \%$ for AKI prediction in our testing dataset. Certain indices like blood gas, serum lactate, cardiac enzymes, and drug levels should increase in frequency and importance toward the onset of AKI, since AKI serves as a marker of clinical deterioration from nosocomial infections, decompensated cardiovascular diseases, major surgery, or nephrotoxicity $[33,34]$. Varying significance of these time-sensitive features in association with evolving AKI may be seen among subsets of patients with sepsis, cardiac failure, or cardiac surgery [35-37]. Our TITV module can provide patient-level interpretation of the feature importance, as suggested by our representative interpretation results in unique AKI case examples (Figure 5). These could provide insightful patient-specific trends to aid the evaluation of AKI etiology [17].

\section{Strengths and Limitations}

Our study has several strengths but is not without limitations. We have studied a large and diverse population with a comprehensive range of medical and surgical conditions not confined to critical care, which improves the generalizability of our analytic module to hospital practice. We excluded patients with more advanced chronic kidney disease, and our $4 \%$ incidence of AKI in the hospital was lower than the $8 \%$ reported in prior studies that used similar EHR methods [33,38]. The lack of precise urine output in ward patients could reduce the model accuracy, but oliguria often develops in 24 hours proximate to AKI and may not fulfill our requirement for a 48-hour prediction window. We have normalized the variables for standardized comparison across different tests. Our novel TITV module provided fine-grained interpretability of the prediction results and achieved accurate prediction simultaneously; this facilitates high-quality health care analytics. Being single center in nature, our AKI prediction module needs to be applied and validated in external health care systems to demonstrate reproducibility. The prediction algorithm could be ported to run on platforms that use similar EHR data architecture, but this naturally limits its deployment to institutions with available technology. Nevertheless, our model could be applied for rolling AKI predictions daily if coupled with a real-time feed of laboratory data. While forward application of the algorithm would naturally encounter model degradation due to concept drift, novel techniques could achieve concept drift detection, understanding, and further adaption from contemporaneous data [39,40]. Furthermore, our algorithm was based on laboratory test results less subjected to case-mix shift over time as compared with disease diagnoses or medication records [41]. We had used zero imputation for missing data, unlike the previously described method of imputing preexisting values in time or median value [38]; zero imputation has been widely adopted in machine learning techniques and has achieved state-of-the-art performance in analytics $[42,43]$. Finally, the subcohort with "false-positive AKI" might be analogous to that of patients with subclinical AKI that may also be associated with adverse long-term outcomes; these were not explored in our study. 


\section{Conclusions}

We have presented a feasible and enhanced EHR analytic module that captures time-sensitive interactions in laboratory investigations and predicts hospital-acquired AKI by a 48-hour lead time. The AKI prediction threshold could be varied to allow the clinically relevant balance in model precision, recall, and predicted AKI numbers that are compatible with patient service load in health care institutions. With a compromised precision in favor of the better recall, our model serves to risk stratify ward patients for detailed clinical or biomarker assessment for true AKI risk. Its real-time deployment is expected to greatly facilitate our upstream efforts to prevent AKI or its complications in hospitalized patients.

\section{Acknowledgments}

This study was supported by a grant from Artificial Intelligence Singapore (100 Experiments Grant), a program supported by the National Research Foundation and hosted by the National University of Singapore. The granting body was not involved in the review or approval of the manuscript for publication. The publication was supported by the Data and Technology Office, National University Health System, Singapore. We thank our institution's EHR data managers, Mr Khong-Hai Wang and Mr Mohammad Shaheryar Furqan, and administrator, Ms Mar Mar Win, for their invaluable support.

\section{Authors' Contributions}

HRC, AV, KYN, and HYT conceived the study concept. HRC, KZ, KYN, KA, and BCO planned the methodology and obtained the ethics approval. HRC, KZ, KYN, KA, and BCO retrieved the EHRs. HRC, KZ, AV, HKY, LL, HYT, AM, GM, and SLL preprocessed and curated the data. $\mathrm{KZ}$ and $\mathrm{BCO}$ applied the machine learning techniques and analyzed the data. HRC, KZ, AV, HKY, LL, HYT, AM, GM, and SLL performed the interim review of analytics. HRC, KZ, and BCO wrote the manuscript. All authors were involved in revising the manuscript and take responsibility for the data presented.

\section{Conflicts of Interest}

None declared.

\section{References}

1. Fabbian F, Savriè C, De Giorgi A, Cappadona R, Di Simone E, Boari B, et al. Acute kidney injury and in-hospital mortality: a retrospective analysis of a nationwide administrative database of elderly subjects in Italy. J Clin Med 2019 Sep 02;8(9):1 [FREE Full text] [doi: 10.3390/jcm8091371] [Medline: 31480750]

2. Christiansen CF, Johansen MB, Langeberg WJ, Fryzek JP, Sørensen HT. Incidence of acute kidney injury in cancer patients: a Danish population-based cohort study. Eur J Intern Med 2011 Aug;22(4):399-406. [doi: 10.1016/j.ejim.2011.05.005] [Medline: 21767759]

3. Pavkov ME, Harding JL, Burrows NR. Trends in hospitalizations for acute kidney injury_United States, $2000-2014$. MMWR Morb Mortal Wkly Rep 2018 Mar 16;67(10):289-293 [FREE Full text] [doi: 10.15585/mmwr.mm6710a2] [Medline: 29543788]

4. Low S, Vathsala A, Murali TM, Pang L, MacLaren G, Ng W, et al. Electronic health records accurately predict renal replacement therapy in acute kidney injury. BMC Nephrol 2019 Jan 31;20(1):32 [FREE Full text] [doi:

10.1186/s12882-019-1206-4] [Medline: 30704418]

5. Bagshaw SM, Uchino S, Bellomo R, Morimatsu H, Morgera S, Schetz M, Beginning and Ending Supportive Therapy for the Kidney (BEST Kidney) Investigators. Septic acute kidney injury in critically ill patients: clinical characteristics and outcomes. Clin J Am Soc Nephrol 2007 May;2(3):431-439 [FREE Full text] [doi: 10.2215/CJN.03681106] [Medline: 17699448]

6. Ong L, Tambyah PA, Lum LH, Low Z, Cheng I, Murali TM, et al. Aminoglycoside-associated acute kidney injury in elderly patients with and without shock. J Antimicrob Chemother 2016 Nov;71(11):3250-3257. [doi: 10.1093/jac/dkw296] [Medline: $\underline{27494924]}$

7. Da Y, Akalya K, Murali T, Vathsala A, Tan C, Low S, et al. Serial quantification of urinary protein biomarkers to predict drug-induced acute kidney injury. Curr Drug Metab 2019;20(8):656-664. [doi: 10.2174/1389200220666190711114504] [Medline: $\underline{\text { 31296157] }}$

8. Chong E, Poh KK, Liang S, Soon CY, Tan H. Comparison of risks and clinical predictors of contrast-induced nephropathy in patients undergoing emergency versus nonemergency percutaneous coronary interventions. J Interv Cardiol 2010 Oct;23(5):451-459 [FREE Full text] [doi: 10.1111/j.1540-8183.2010.00581.x] [Medline: 20796168]

9. Chong E, Poh K, Lu Q, Zhang JJ, Tan N, Hou XM, et al. Comparison of combination therapy of high-dose oral $\mathrm{N}$-acetylcysteine and intravenous sodium bicarbonate hydration with individual therapies in the reduction of Contrast-induced Nephropathy during Cardiac Catheterisation and Percutaneous Coronary Intervention (CONTRAST): a multi-centre, randomised, controlled trial. Int J Cardiol 2015 Dec 15;201:237-242. [doi: 10.1016/j.ijcard.2015.07.108] [Medline: 26301645]

10. Chua H, Wong W, Ong VH, Agrawal D, Vathsala A, Tay H, et al. Extended mortality and chronic kidney disease after septic acute kidney injury. J Intensive Care Med 2020 Jun;35(6):527-535. [doi: 10.1177/0885066618764617] [Medline: 29552953] 
11. Hong W, Haroon S, Lau T, Hui S, Ma V, Goh J, et al. Transitional care program to facilitate recovery following severe acute kidney injury. J Nephrol 2019 Aug;32(4):605-613. [doi: 10.1007/s40620-019-00616-z] [Medline: 31090023]

12. Choi E, Bahadori MT, Schuetz A, Stewart WF, Sun J. Doctor AI: predicting clinical events via recurrent neural networks. JMLR Workshop Conf Proc 2016 Aug;56:301-318 [FREE Full text] [Medline: 28286600]

13. Wonnacott A, Meran S, Amphlett B, Talabani B, Phillips A. Epidemiology and outcomes in community-acquired versus hospital-acquired AKI. Clin J Am Soc Nephrol 2014 Jun 06;9(6):1007-1014 [FREE Full text] [doi: 10.2215/CJN.07920713] [Medline: 24677557]

14. Willis K, Cheung M, Slifer S. KDIGO 2012 Clinical Practice Guideline for the Evaluation and Management of Chronic Kidney Disease. Kidney Disease: Improving Global Outcomes. 2013. URL: https://kdigo.org/wp-content/uploads/2017/ 02/KDIGO_2012_CKD_GL.pdf [accessed 2021-12-02]

15. Levey AS, Stevens LA, Schmid CH, Zhang YL, Castro AF, Feldman HI, CKD-EPI (Chronic Kidney Disease Epidemiology Collaboration). A new equation to estimate glomerular filtration rate. Ann Intern Med 2009 May 05;150(9):604-612 [FREE Full text] [doi: 10.7326/0003-4819-150-9-200905050-00006] [Medline: 19414839]

16. Khwaja A. KDIGO clinical practice guidelines for acute kidney injury. Nephron Clin Pract 2012;120(4):c179-c184 [FREE Full text] [doi: 10.1159/000339789] [Medline: 22890468]

17. Zheng K, Cai S, Chua H, Wang W, Ngiam K, Ooi B. Tracer: a framework for facilitating accurate and interpretable analytics for high stakes applications. Proc 2020 ACM SIGMOD Int Conf Manag Data 2020:1747-1763. [doi:

10.1145/3318464.3389720]

18. Choi E, Bahadori M, Kulas J, Schuetz A, Stewart W, Sun J. Retain: an interpretable predictive model for healthcare using reverse time attention mechanism. Proc 30th Int Conf Neural Inf Proc Syst 2016:3512-3520. [doi: 10.5555/3157382.3157490]

19. Ma F, Chitta R, Zhou J, You Q, Sun T, Gao J. Dipole: diagnosis prediction in healthcare via attention-based bidirectional recurrent neural networks. ArXiv. Preprint posted online on June 19, 2017 2017:1903-1911 [FREE Full text] [doi: 10.1145/3097983.3098088]

20. Bai T, Egleston BL, Zhang S, Vucetic S. Interpretable representation learning for healthcare via capturing disease progression through time. KDD 2018 Aug:43-51 [FREE Full text] [doi: 10.1145/3219819.3219904] [Medline: 31037221]

21. kaiping/TRACER. URL: https://github.com/kaiping/TRACER [accessed 2021-12-02]

22. Liu KD, Goldstein SL, Vijayan A, Parikh CR, Kashani K, Okusa MD, AKI!Now Initiative of the American Society of Nephrology, et al. AKI!Now initiative: recommendations for awareness, recognition, and management of AKI. Clin J Am Soc Nephrol 2020 Dec 07;15(12):1838-1847. [doi: 10.2215/CJN.15611219] [Medline: 32317329]

23. Kashani K, Al-Khafaji A, Ardiles T, Artigas A, Bagshaw SM, Bell M, et al. Discovery and validation of cell cycle arrest biomarkers in human acute kidney injury. Crit Care 2013 Mar 06;17(1):R25 [FREE Full text] [doi: 10.1186/cc12503] [Medline: 23388612]

24. Rank N, Pfahringer B, Kempfert J, Stamm C, Kühne T, Schoenrath F, et al. Deep-learning-based real-time prediction of acute kidney injury outperforms human predictive performance. NPJ Digit Med 2020;3:139 [FREE Full text] [doi: 10.1038/s41746-020-00346-8] [Medline: 33134556]

25. Tomašev N, Glorot X, Rae JW, Zielinski M, Askham H, Saraiva A, et al. A clinically applicable approach to continuous prediction of future acute kidney injury. Nature 2019 Aug;572(7767):116-119. [doi: 10.1038/s41586-019-1390-1] [Medline: 31367026]

26. Meersch M, Schmidt C, Hoffmeier A, Van Aken H, Wempe C, Gerss J, et al. Prevention of cardiac surgery-associated AKI by implementing the KDIGO guidelines in high risk patients identified by biomarkers: the PrevAKI randomized controlled trial. Intensive Care Med 2017 Nov;43(11):1551-1561 [FREE Full text] [doi: 10.1007/s00134-016-4670-3] [Medline: 28110412]

27. Self WH, Semler MW, Wanderer JP, Wang L, Byrne DW, Collins SP, SALT-ED Investigators. Balanced crystalloids versus saline in noncritically ill adults. N Engl J Med 2018 Mar 01;378(9):819-828 [FREE Full text] [doi: 10.1056/NEJMoa1711586] [Medline: 29485926]

28. Goldstein SL, Mottes T, Simpson K, Barclay C, Muething S, Haslam DB, et al. A sustained quality improvement program reduces nephrotoxic medication-associated acute kidney injury. Kidney Int 2016 Dec;90(1):212-221. [doi: 10.1016/j.kint.2016.03.031] [Medline: 27217196]

29. Kolhe NV, Reilly T, Leung J, Fluck RJ, Swinscoe KE, Selby NM, et al. A simple care bundle for use in acute kidney injury: a propensity score-matched cohort study. Nephrol Dial Transplant 2016 Nov;31(11):1846-1854. [doi: 10.1093/ndt/gfw087] [Medline: 27190331]

30. Selby NM, Casula A, Lamming L, Stoves J, Samarasinghe Y, Lewington AJ, et al. An organizational-level program of intervention for AKI: a pragmatic stepped wedge cluster randomized trial. J Am Soc Nephrol 2019 Mar;30(3):505-515 [FREE Full text] [doi: 10.1681/ASN.2018090886] [Medline: 31058607]

31. Jones RG, Johnson OA, Batstone G. Informatics and the clinical laboratory. Clin Biochem Rev 2014 Aug;35(3):177-192 [FREE Full text] [Medline: 25336763]

32. Cheng P, Waitman LR, Hu Y, Liu M. Predicting inpatient acute kidney injury over different time horizons: how early and accurate? AMIA Annu Symp Proc 2017;2017:565-574 [FREE Full text] [Medline: 29854121] 
33. Jannot A, Burgun A, Thervet E, Pallet N. The diagnosis-wide landscape of hospital-acquired AKI. Clin J Am Soc Nephrol 2017 Jun 07;12(6):874-884 [FREE Full text] [doi: 10.2215/CJN.10981016] [Medline: 28495862]

34. Sykes L, Sinha S, Hegarty J, Flanagan E, Doyle L, Hoolickin C, et al. Reducing acute kidney injury incidence and progression in a large teaching hospital. BMJ Open Qual 2018;7(4):e000308 [FREE Full text] [doi: 10.1136/bmjoq-2017-000308] [Medline: 30555931$]$

35. Plataki M, Kashani K, Cabello-Garza J, Maldonado F, Kashyap R, Kor DJ, et al. Predictors of acute kidney injury in septic shock patients: an observational cohort study. Clin J Am Soc Nephrol 2011 Jul;6(7):1744-1751 [FREE Full text] [doi: 10.2215/CJN.05480610] [Medline: 21734090]

36. Holgado JL, Lopez C, Fernandez A, Sauri I, Uso R, Trillo JL, et al. Acute kidney injury in heart failure: a population study. ESC Heart Fail 2020 Apr;7(2):415-422 [FREE Full text] [doi: 10.1002/ehf2.12595] [Medline: 32059081]

37. Ng RRG, Chew STH, Liu W, Shen L, Ti LK. Identification of modifiable risk factors for acute kidney injury after coronary artery bypass graft surgery in an Asian population. J Thorac Cardiovasc Surg 2014 Apr;147(4):1356-1361 [FREE Full text] [doi: 10.1016/j.jtcvs.2013.09.040] [Medline: 24183907]

38. Koyner JL, Adhikari R, Edelson DP, Churpek MM. Development of a multicenter ward-based AKI prediction model. Clin J Am Soc Nephrol 2016 Nov 07;11(11):1935-1943 [FREE Full text] [doi: 10.2215/CJN.00280116] [Medline: 27633727]

39. Lu J, Liu A, Dong F, Gu F, Gama J, Zhang G. Learning under concept drift: a review. IEEE Trans Knowl Data Eng 2019;31(12):2346-2363. [doi: 10.1109/tkde.2018.2876857]

40. Gama J, Žliobaitė I, Bifet A, Pechenizkiy M, Bouchachia A. A survey on concept drift adaptation. ACM Comput Surv 2014 Apr;46(4):1-37. [doi: 10.1145/2523813]

41. Davis SE, Lasko TA, Chen G, Siew ED, Matheny ME. Calibration drift in regression and machine learning models for acute kidney injury. J Am Med Inform Assoc 2017 Nov 01;24(6):1052-1061 [FREE Full text] [doi: 10.1093/jamia/ocx030] [Medline: 28379439]

42. Zheng K, Gao J, Ngiam K, Ooi B, Yip W. Resolving the bias in electronic medical records. Proc 23rd ACM SIGKDD Int Con Knowl Discov Data Min 2017:2171-2180. [doi: 10.1145/3097983.3098149]

43. Zheng Y, Tang B, Ding W, Zhou H. A neural autoregressive approach to collaborative filtering. Proc 33rd Int Conf Int Conf Mach Learn 2016:764-773. [doi: 10.5555/3045390.3045472]

\section{Abbreviations \\ AKI: acute kidney injury \\ AUC: area under the receiver operating characteristic curve \\ eGFR: estimated glomerular filtration rate \\ EHR: electronic health record \\ KDIGO: Kidney Disease: Improving Global Outcomes \\ TITV: time-invariant and time-variant}

Edited by R Kukafka; submitted 30.05.21; peer-reviewed by Z Ren, M Liu, E Sükei; comments to author 02.08.21; revised version
received 26.09.21; accepted 29.10.21; published 24.12.21
Please cite as:
Chua HR, Zheng K, Vathsala A, Ngiam KY, Yap HK, Lu L, Tiong HY, Mukhopadhyay A, MacLaren G, Lim SL, Akalya K, Ooi BC
Health Care Analytics With Time-Invariant and Time-Variant Feature Importance to Predict Hospital-Acquired Acute Kidney Injury:
Observational Longitudinal Study
J Med Internet Res 2021;23(12):e30805
URL: https://www.jmir.org/2021/12/e30805
doi: $10.2196 / 30805$
PMID:

CHorng-Ruey Chua, Kaiping Zheng, Anantharaman Vathsala, Kee-Yuan Ngiam, Hui-Kim Yap, Liangjian Lu, Ho-Yee Tiong, Amartya Mukhopadhyay, Graeme MacLaren, Shir-Lynn Lim, K Akalya, Beng-Chin Ooi. Originally published in the Journal of Medical Internet Research (https://www.jmir.org), 24.12.2021. This is an open-access article distributed under the terms of the Creative Commons Attribution License (https://creativecommons.org/licenses/by/4.0/), which permits unrestricted use, distribution, and reproduction in any medium, provided the original work, first published in the Journal of Medical Internet Research, is properly cited. The complete bibliographic information, a link to the original publication on https://www.jmir.org/, as well as this copyright and license information must be included. 